\title{
FORMATION AND PLASMA CIRCULATION OF SOLAR PROMINENCES
}

\author{
C. XIA ${ }^{1}$ and R. KePPENS ${ }^{1,2}$ \\ ${ }^{1}$ Centre for mathematical Plasma Astrophysics, Department of Mathematics, KU Leuven, Celestijnenlaan 200B, B-3001 Leuven, Belgium \\ ${ }^{2}$ School of Astronomy and Space Science, Nanjing University, Nanjing 210093, China \\ Received 2016 February 19; accepted 2016 March 16; published 2016 May 17
}

\begin{abstract}
Solar prominences are long-lived cool and dense plasma curtains in the hot and rarefied outer solar atmosphere or corona. The physical mechanism responsible for their formation and especially for their internal plasma circulation has been uncertain for decades. The observed ubiquitous downflows in quiescent prominences are difficult to interpret because plasma with high conductivity seems to move across horizontal magnetic field lines. Here we present three-dimensional numerical simulations of prominence formation and evolution in an elongated magnetic flux rope as a result of in situ plasma condensations fueled by continuous plasma evaporation from the solar chromosphere. The prominence is born and maintained in a fragmented, highly dynamic state with continuous reappearance of multiple blobs and thread structures that move mainly downward, dragging along mass-loaded field lines. The circulation of prominence plasma is characterized by the dynamic balance between the drainage of prominence plasma back to the chromosphere and the formation of prominence plasma via continuous condensation. Plasma evaporates from the chromosphere, condenses into the prominence in the corona, and drains back to the chromosphere, establishing a stable chromosphere-corona plasma cycle. Synthetic images of the modeled prominence with the Solar Dynamics Observatory Atmospheric Imaging Assembly closely resemble actual observations, with many dynamical threads underlying an elliptical coronal cavity.
\end{abstract}

Key words: magnetohydrodynamics (MHD) - Sun: corona - Sun: filaments, prominences

Supporting material: animations

\section{INTRODUCTION}

Solar prominences are about 100 times denser and cooler than their surrounding hot corona, with their weight supported by the magnetic field against gravity. They exist for days to weeks above polarity inversion lines (PILs), which separate positive and negative magnetic flux regions in the photosphere. Prominences are frequently found inside coronal cavities, which are tunnel-like elliptical dark regions (Gibson et al. 2010) at the solar limb, with up to $40 \%$ density depletion (Fuller et al. 2008), around the prominences. The magnetic structure hosting a prominence and its cavity is believed to be a helical magnetic flux rope confined by overlying magnetic loops, which is consistent with observed spinning plasma motions (Wang \& Stenborg 2010) as well as linear polarization signatures (Bak-Stęślicka et al. 2013). A flux rope was present in early two-dimensional (2D) theoretical models of prominences (Kuperus \& Raadu 1974), and 2D magnetohydrostatic solutions for prominence-embedded flux ropes have been demonstrated analytically (Low \& Zhang 2004) and numerically (Blokland \& Keppens 2011; Hillier \& van Ballegooijen 2013).

Static models of prominences assume that quiescent prominences change little with time. However, high-resolution observations have found that quiescent prominences consist of fine, vertically oriented plasma threads, which evolve continuously with downward motions $\left(4-35 \mathrm{~km} \mathrm{~s}^{-1}\right)$ (Engvold 1976; Berger et al. 2008; Chae et al. 2008). Moreover, episodic dark plumes may rise (Berger et al. 2008, 2011) from the bottom of prominences and propagate upward between threads at speeds of about $10-20 \mathrm{~km} \mathrm{~s}^{-1}$. The heavy prominence material suspended above the light coronal plasma can be liable to Rayleigh-Taylor (RT) instability (Ryutova et al. 2010), which has been investigated in several numerical magnetohydrodynamic (MHD) models (Hillier et al. 2011; Khomenko et al. 2014; Keppens et al. 2015; Terradas et al. 2015). However, none of these models addressed prominence formation and mass cycling, or studied a realistic 3D flux rope.

The idea that prominences form via in situ condensation of coronal plasma was inferred from thermal instability theory (Parker 1953; Field 1965) and is supported by observations in extreme ultraviolet (EUV) channels (Berger et al. 2012; Liu et al. 2012). Moreover, continuous condensations must happen to compensate mass loss of quiescent prominences, which requires a continuous supply of hot plasma from chromosphere to corona. Such hot plasma flows have not been convincingly detected, although persistent horizontal flows of $\mathrm{H} \alpha$-emitting cool plasma originating from the chromosphere into a prominence were observed (Chae et al. 2008), and the episodic dark rising plumes filled with hot plasma may provide a small fraction of the required mass (Berger et al. 2010). Numerical modeling of condensations as a result of runaway radiative cooling started with one-dimensional hydrodynamic simulations along individual magnetic field lines (Antiochos et al. 1999; Karpen et al. 2001; Xia et al. 2011; Luna et al. 2012) and progressed in recent years to 2D MHD simulations in magnetic arcades (Xia et al. 2012; Keppens \& Xia 2014). These models add localized heating at footpoints of magnetic loops to evaporate plasma from chromosphere to corona, leading to continuous condensations. However, restricted by $1 \mathrm{D}$ or $2 \mathrm{D}$ setups, they cannot fully establish the internal dynamics of prominences. Recent work on prominence formation via in situ condensation in a 2D (Kaneko \& Yokoyama 2015) and 3D flux rope (Xia et al. 2014a) did not reproduce the internal dynamics and mass drainage, and found only a small prominence fragment that settled in a magnetostatic equilibrium. 
We here report a set of 3D models that for the first time ever demonstrate continuous formation of prominence condensations in a coronal cavity, achieving a balanced chromospherecorona plasma cycle characterized by vertical flows in thin prominence threads. The numerical methods and the simulation procedure are explained in Section 2. We present the results in Section 3. Finally, the results are discussed in Section 4.

\section{SIMULATION STRATEGY}

Since the magnetic topology of quiescent prominences is believed to be an elongated magnetic flux rope, we first perform isothermal MHD simulations to simulate the formation of a magnetic flux rope driven by footpoint motions and flux cancellation (van Ballegooijen \& Martens 1989; Xia et al. 2014b). Our 3D model is set up in a Cartesian box covering $200 \mathrm{Mm} \times 120 \mathrm{Mm} \times 80 \mathrm{Mm}$ in $x(-100 \mathrm{Mm}$ to $100 \mathrm{Mm}), y(-60 \mathrm{Mm}$ to $60 \mathrm{Mm})$, and $z(0-80 \mathrm{Mm})$ directions, where a finite beta corona at a constant temperature of $1 \mathrm{MK}$ is initially constructed with density and gas pressure prescribed from hydrostatic equilibrium with a number density at the bottom of $10^{9} \mathrm{~cm}^{-3}$. We start from a bipolar magnetic field in the shape of an elongated sheared arcade. This arcade field itself is generated by linear force-free field extrapolation from an analytically prescribed bipolar magnetogram with two regions of opposite polarity shaped like two parallel baguettes. This magnetogram $B_{m}$ is formulated as

$$
B_{m}=\left\{\begin{array}{c}
B_{0} \exp \left(-\frac{\left(x-x_{1}+x_{L}\right)^{2}}{2 \delta x^{2}}-\frac{\left(y-y_{1}\right)^{2}}{2 \delta y^{2}}\right) \\
\quad-B_{0} \exp \left(-\frac{\left(x-x_{2}+x_{L}\right)^{2}}{2 \delta x^{2}}-\frac{\left(y-y_{2}\right)^{2}}{2 \delta y^{2}}\right) \\
\text { if } x<x_{1}-x_{L} \\
B_{0} \exp \left(-\frac{\left(y-y_{1}\right)^{2}}{2 \delta y^{2}}\right)-B_{0} \exp \left(-\frac{\left(y-y_{2}\right)^{2}}{2 \delta y^{2}}\right) \\
\quad \text { if } x_{1}-x_{L} \leqslant x \leqslant x_{1}+x_{L} \\
B_{0} \exp \left(-\frac{\left(x-x_{1}-x_{L}\right)^{2}}{2 \delta x^{2}}-\frac{\left(y-y_{1}\right)^{2}}{2 \delta y^{2}}\right) \\
\quad-B_{0} \exp \left(-\frac{\left(x-x_{2}-x_{L}\right)^{2}}{2 \delta x^{2}}-\frac{\left(y-y_{2}\right)^{2}}{2 \delta y^{2}}\right) \\
\text { if } x>x_{1}+x_{L}
\end{array}\right.
$$

where $B_{0}=20 \mathrm{G}, \quad x_{1}=x_{2}=0 \mathrm{Mm}, \quad y_{1}=-y_{2}=25 \mathrm{Mm}$, $x_{L}=50 \mathrm{Mm}, \delta x=10 \mathrm{Mm}$, and $\delta y=13 \mathrm{Mm}$. An exact Green's function method (Chiu \& Hilton 1977) is used to generate an initial linear force-free field with constant $\alpha=-0.08$. Since our simulation box is intended to start at low chromospheric heights, this magnetogram is placed $4 \mathrm{Mm}$ below the bottom plane of the simulation box.

A composite surface flow is imposed at the bottom boundary with the formula

$$
v_{y}^{b}=-f(t) C_{1} \frac{\partial\left|B_{m}\right|}{\partial y} \exp \left(-y^{2} / y_{d}^{2}\right), \quad v_{x}^{b}=-v_{y}^{b}
$$

where $y_{d}=35 \mathrm{Mm}$ quantifies an additional Gaussian width parameter away from the PIL, $f(t)$ is a linear ramp function to switch the driving flow on and off, and the amplitude factor $C_{1}$ is chosen so that the driving speed has a maximum value of $12.8 \mathrm{~km} \mathrm{~s}^{-1}$ and the maximum initial Alfvén Mach number is 0.0155 . The flow is a combination of a shearing flow and a converging flow relative to the PIL. The shearing flow roughly mimics the effective shearing of an east-west bipolar arcade by the differential rotation of the solar surface. The converging flow from the regions of strong field to regions of weak field is an effective result of magnetic element diffusion caused by random supergranular motions (Leighton 1964). Flows far away from the PIL are suppressed by the exponential factor for an easier handling of the side boundaries.

The model evolution is performed by solving the isothermal MHD equations given by

$$
\begin{gathered}
\frac{\partial \rho}{\partial t}+\nabla \cdot(\rho \boldsymbol{v})=0, \\
\frac{\partial(\rho \boldsymbol{v})}{\partial t}+\nabla \cdot\left(\rho \boldsymbol{v} \boldsymbol{v}+p_{\mathrm{tot}} \boldsymbol{I}-\frac{\boldsymbol{B} \boldsymbol{B}}{\mu_{0}}\right)=\rho \boldsymbol{g}, \\
\frac{\partial \boldsymbol{B}}{\partial t}+\nabla \cdot(\boldsymbol{v} \boldsymbol{B}-\boldsymbol{B} \boldsymbol{v})=0,
\end{gathered}
$$

where $\rho, \boldsymbol{v}, \boldsymbol{B}$, and $\boldsymbol{I}$ are the plasma density, velocity, magnetic field, and unit tensor, respectively, while the total pressure is $p_{\text {tot }} \equiv p+\frac{B^{2}}{2 \mu_{0}}$ and $\boldsymbol{g}=-g_{\odot} r_{\odot}^{2} /\left(r_{\odot}+z\right)^{2} \hat{z}$ is the gravitational acceleration with $r_{\odot}$ the solar radius and $g_{\odot}$ the gravitational acceleration at the solar surface. To normalize the equations for computation, we use $10 \mathrm{Mm}, 10^{9} \mathrm{~cm}^{-3}, 116.45 \mathrm{~km} \mathrm{~s}^{-1}$, and $2 \mathrm{G}$ as the units of length, number density, velocity, and magnetic field, respectively. We use the adaptive mesh refinement (AMR) versatile advection code (MPI-AMRVAC) (Keppens et al. 2012; Porth et al. 2014) to numerically solve these equations with a third-order accurate scheme combining a Harten-Lax-van Leer scheme (Harten et al. 1983) with a thirdorder limited reconstruction (Čada \& Torrilhon 2009) and a three-step Runge-Kutta time integration. To numerically maintain the divergence of the magnetic field close to zero, we use the generalized Lagrangian multiplier method (Dedner et al. 2002). The model domain at this phase is discretized on a three-level AMR grid that has an effective resolution of $400 \times 240 \times 240$ with the smallest cells of $500 \mathrm{~km} \times 500 \mathrm{~km}$ $\times 333 \mathrm{~km}$. The simulation setup is completed by the following boundary conditions. We force zero vertical velocity on the bottom face and zero velocity on the other five faces of the box by antisymmetric boundary conditions. We extrapolate the magnetic field at the bottom boundary and the top boundary from inner physical values, keeping the normal gradient zero, with a one-sided third-order finite difference representation. Then we modify the normal component to enforce the divergence-free condition in a second-order centered difference evaluation. The magnetic fields at the four side boundaries are kept fixed. The density values in the cells of the side boundary are copied from the neighboring cells of the physical domain. We fix the gravitationally stratified density at the bottom and adopt a gravitationally stratified density profile at the top.

The bottom flows drive those coronal loops that are rooted in the inner halves of the main arcade polarities to move toward aligning with the PIL. As footpoints from opposite polarities collide on the PIL, magnetic reconnections occur due to finite numerical resistivity, to join arched loops into helical loops in a head-to-tail style. These helical loops, winding about the same central axial field line, form a helical magnetic flux rope (see Figure 1). We completely switch off the driving flows after 100 


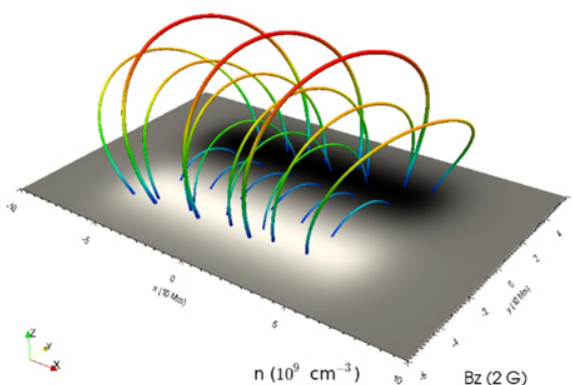

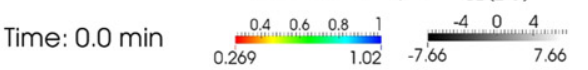

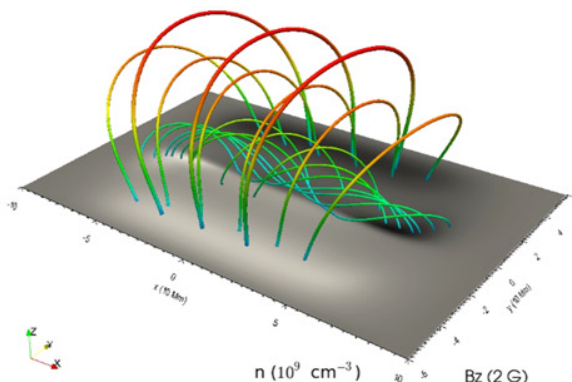

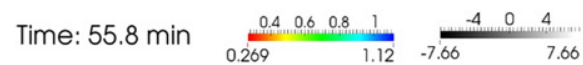

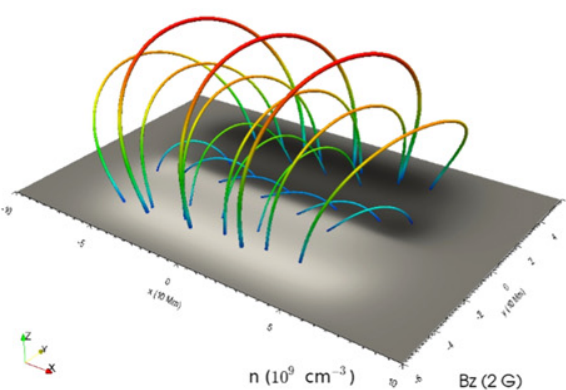

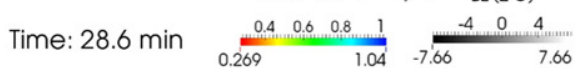

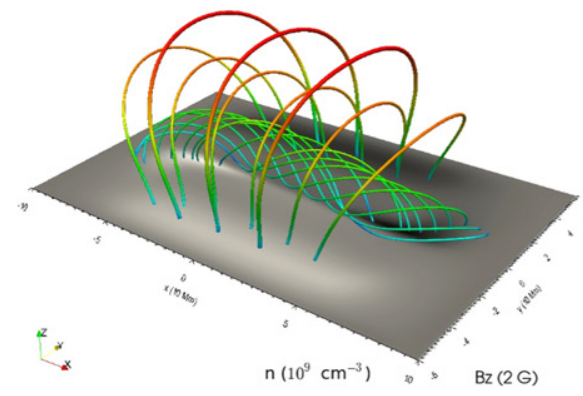

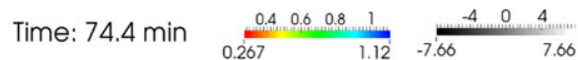

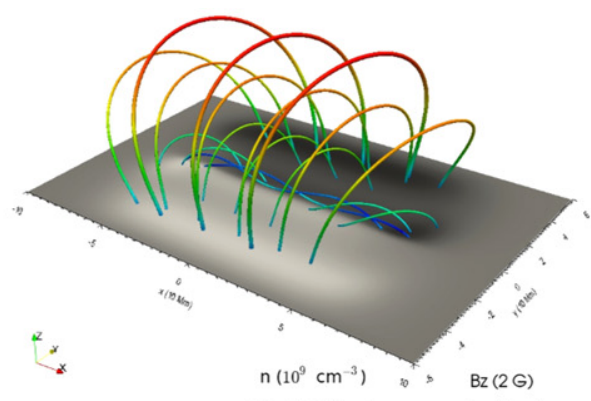

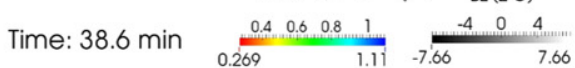

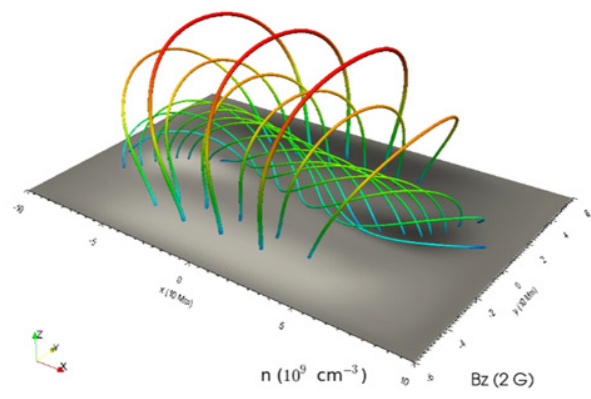

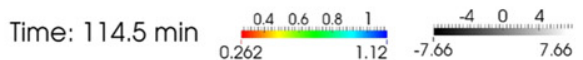

Figure 1. The formation process of a magnetic flux rope in the solar corona. In these panels depicting six subsequent moments, magnetic field lines colored by number density $(n)$ show the forming flux rope and overlying arcade loops, and the grayscale of the bottom plane indicates vertical magnetic field strength.

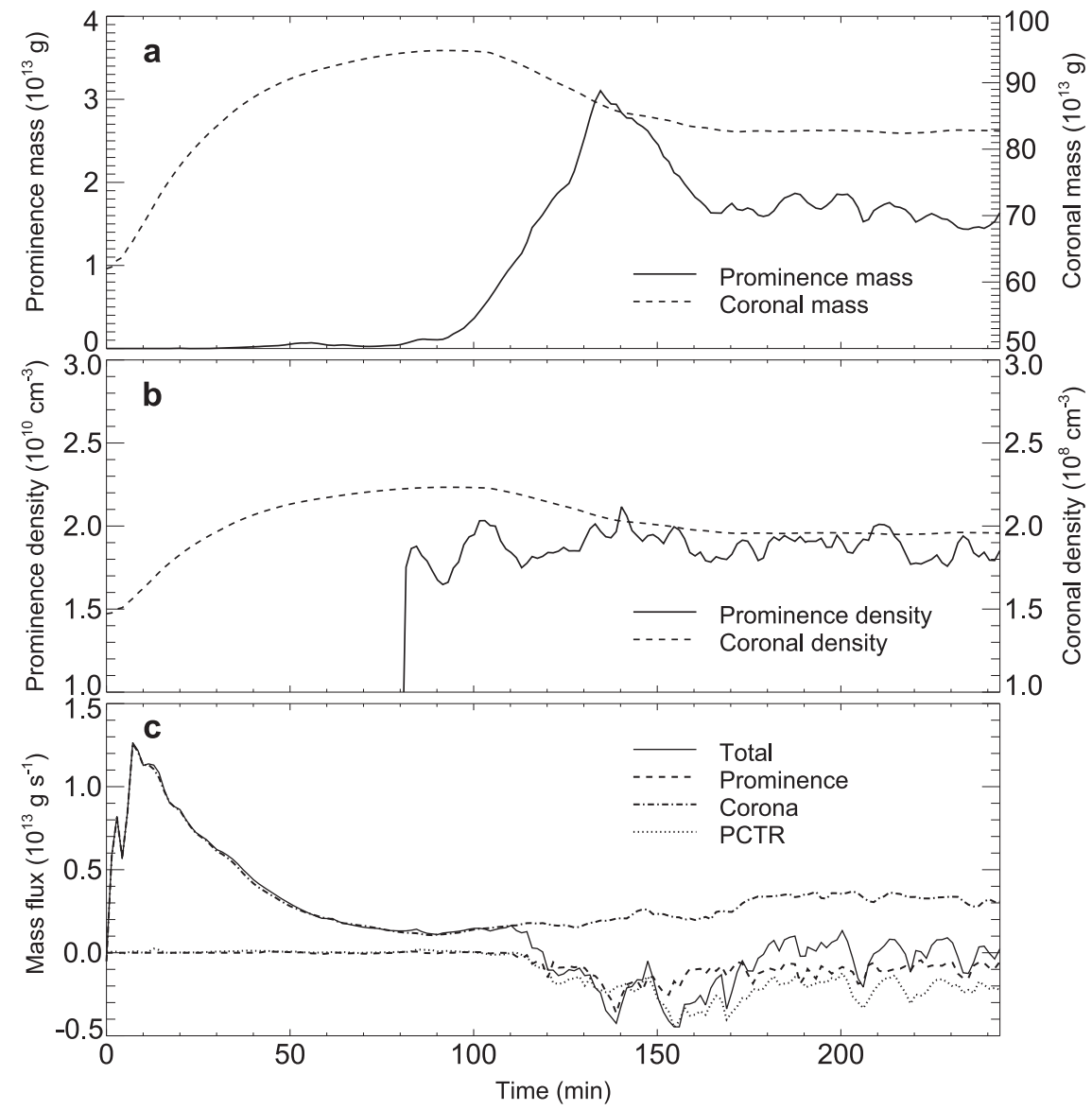

Figure 2. Quantifying the formation and mass circulation of a virtual prominence. (a) Evolution of prominence mass (solid line) and coronal mass (dashed line) in the box. (b) Evolution of average plasma number density of the prominence (solid line) and the corona (dashed line). (c) Evolution of mass flux, through the bottom coronal plane $(z=8 \mathrm{Mm})$, of prominence plasma (dashed line), coronal plasma (dashed-dotted line), the prominence-corona transition region (PCTR) (dotted line), and all plasma together (solid line). The PCTR is where the plasma density is lower than $10^{9} \mathrm{~cm}^{-3}$ and the temperature lower than $100,000 \mathrm{~K}$. 


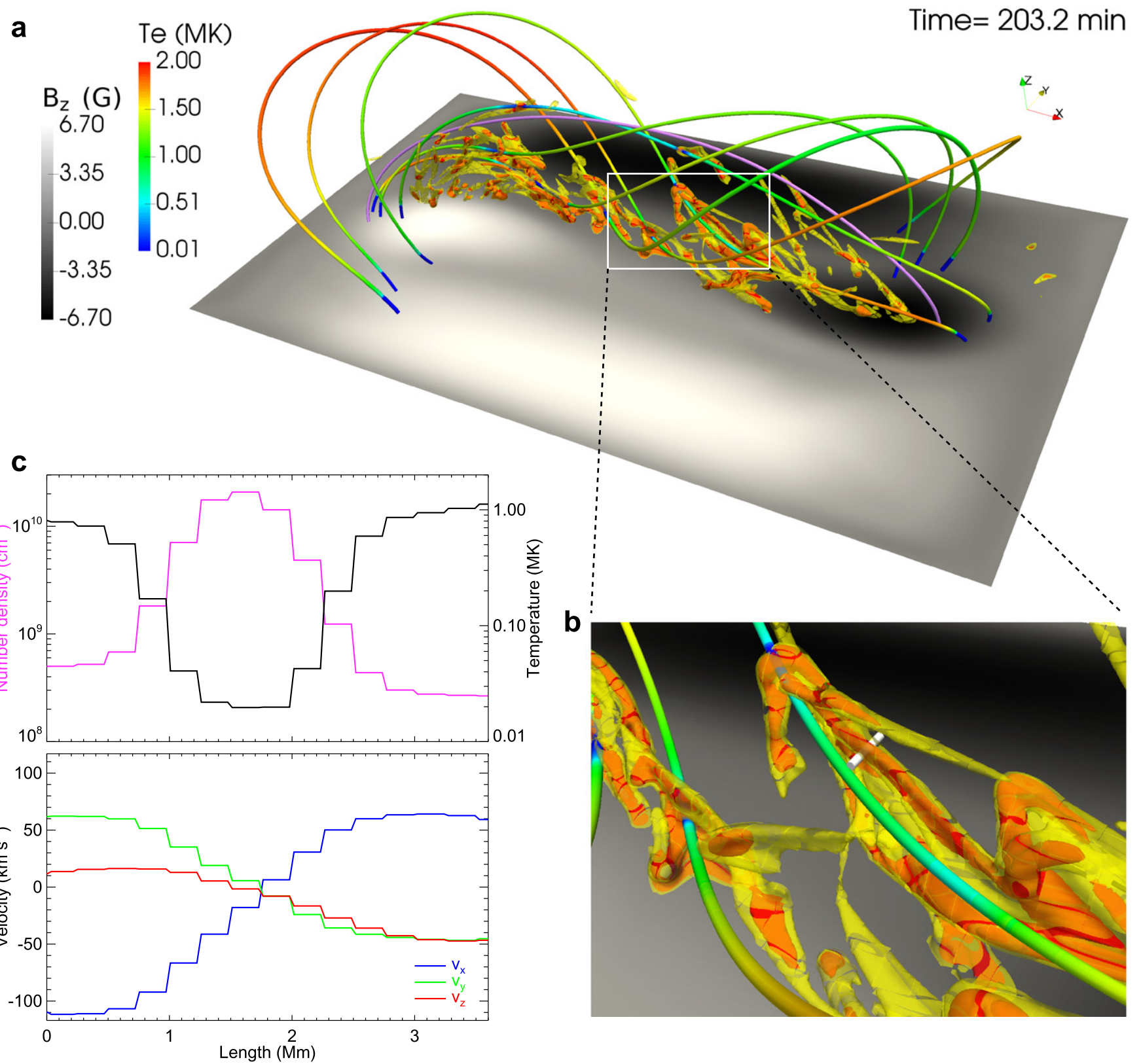

Figure 3. Prominence, magnetic field lines, and shearing flows at a time of 203.2 minutes. (a) A global view of the prominence with the yellow translucent density contours at $4 \times 10^{9} \mathrm{~cm}^{-3}$, the red density contours at $10^{10} \mathrm{~cm}^{-3}$, magnetic field lines (colored by temperature $T e$ ) threading through the prominence, a purple magnetic field line representing the axis of the flux rope, and the bottom plane colored by $B_{z}$. (b) Close-up view of the region in the white rectangular box in (a) with a white straight line cutting through a thread and the number density (pink curve), temperature (black curve), and velocities (blue curve $V_{x}$, green curve $V_{y}$, red curve $V_{z}$ ) along the cutting line displayed in (c).

minutes and let the flux rope relax to an equilibrium state. At the end of this stage, a large-scale elongated flux rope is formed with its flux surface touching the bottom plane. The length of the flux rope is about $160 \mathrm{Mm}$ and the diameter of its cross section is about $34 \mathrm{Mm}$. The density distribution does not change much compared to the initial state and the magnetic field has negligible Lorentz force. The magnetic field strength of the flux rope is about $6-7 \mathrm{G}$, which is consistent with the field strength of a quiescent prominence since spectral polarimetric measurements show that the magnetic field in a quiescent prominence ranges from 3 to $30 \mathrm{G}$ and it is predominantly horizontal, making an acute angle with respect to the axis of the prominence (Leroy et al. 1983; Bommier et al. 1994; Orozco Suárez et al. 2014).
In the second stage, we perform a full MHD simulation to produce a modeled solar atmosphere in thermal equilibrium containing the magnetic flux rope that was generated in the isothermal MHD modeling described above. To set up the initial state based on the isothermal flux rope, we kept the magnetic field unchanged and modified the density and temperature profiles from their isothermal states to a static vertically stratified solar atmosphere including chromosphere, transition region, and corona. We set the region below a height of $2.7 \mathrm{Mm}$ to have a chromospheric temperature $9600 \mathrm{~K}$, and above this height the temperature increases with height in such a way that the vertical thermal conduction flux has a constant value of $2 \times 10^{5} \mathrm{erg} \mathrm{cm}^{-2} \mathrm{~s}^{-1}$. The density is then derived assuming a hydrostatic atmosphere with the number density at 


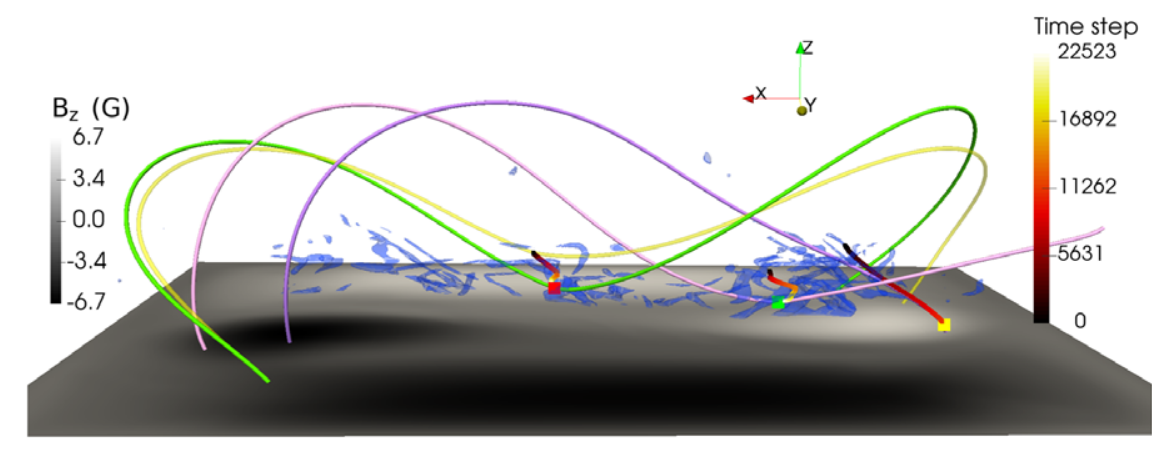

Time $=214.7 \mathrm{~min}$

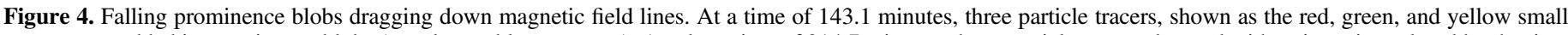

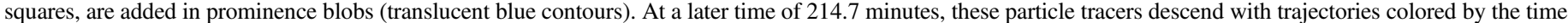

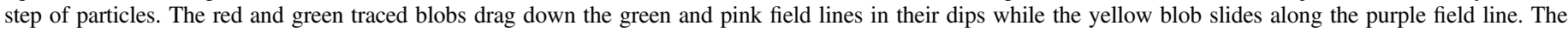
translucent yellow field line is threading through the red tracer at a time of 143.1 minutes.

the bottom being $1.1 \times 10^{13} \mathrm{~cm}^{-3}$. We now consider the full MHD equations with the energy equation as

$$
\begin{aligned}
& \frac{\partial E}{\partial t}+\nabla \cdot\left(E \boldsymbol{v}+p_{\mathrm{tot}} \boldsymbol{v}-\frac{\boldsymbol{B} \boldsymbol{B}}{\mu_{0}} \cdot \boldsymbol{v}\right) \\
& =\rho \boldsymbol{g} \cdot \boldsymbol{v}+H-R+\nabla \cdot(\boldsymbol{\kappa} \cdot \nabla T),
\end{aligned}
$$

where total pressure $p_{\text {tot }} \equiv p+B^{2} / 2 \mu_{0}$, gas pressure $p=2.3 n_{\mathrm{H}} k_{\mathrm{B}} T, \quad$ and total energy $E=p /(\gamma-1)+\rho v^{2} / 2+B^{2} / 2 \mu_{0}$. We again use MPI-AMRVAC with the same combination of schemes as the isothermal model. The field-aligned $\left(\boldsymbol{\kappa}=\kappa_{\|} \hat{\boldsymbol{b}} \hat{\boldsymbol{b}}\right)$ thermal conduction is solved separately using a Runge-Kutta type Super TimeStepping scheme (Meyer et al. 2012), and $\kappa_{\|}=10^{-6} T^{5 / 2} \mathrm{erg} \mathrm{cm}^{-1} \mathrm{~s}^{-1} \mathrm{~K}^{-1}$ is the Spitzer conductivity. Optically thin radiative cooling $R=1.2 n_{\mathrm{H}}^{2} \Lambda(T)$ is added using an exact integration scheme (Townsend 2009). To maintain a hot corona, the coronal heating is simulated by adding the parameterized heating term $H=H_{0}=c_{0} e^{-z / \lambda}$ with $c_{0}=10^{-4} \mathrm{erg} \mathrm{cm}^{-3} \mathrm{~s}^{-1}$ and $\lambda=60 \mathrm{Mm}$. We increased the highest AMR level of the mesh to 4, resulting in an effective resolution of $800 \times 480 \times 480$ with the smallest cells of 250 $\mathrm{km} \times 250 \mathrm{~km} \times 166 \mathrm{~km}$. We modified the boundary conditions from the isothermal model to set zero velocity and fixed magnetic field at all boundaries. The gas pressure is fixed at the bottom and flexible at the top according to gravitational stratification. At the side boundaries, the gas pressure copies values from the inner layer in the physical domain. This modified plasma state is not in thermal equilibrium, so we numerically solve the full MHD equations involving optically thin radiative loss, anisotropic thermal conduction, and coronal heating until the system relaxes to a quasi-equilibrium after 114.5 minutes. Our end-state in this phase is reached when all thermal quantities settle into a stable 3D configuration where the magnitude of the remaining velocity is less than $10 \mathrm{~km} \mathrm{~s}^{-1}$, and it is representative of a stable flux rope configuration with hot coronal plasma trapped inside it, where twisted field lines connect chromospheric to coronal plasma regions.

In the third stage, we aim to form the macroscopic prominence in the stable flux rope obtained in the second stage. In order to simulate the chromospheric evaporation, which is an efficient way to bring material from the chromosphere to the corona, a relatively strong localized heating $H_{1}$ is added to the energy equation in addition to the global coronal heating $H_{0}$, namely, $H=H_{0}+H_{1}$. We only add the localized heating $H_{1}$ in two fixed cylinders that coincide with the two footpoint regions of the flux rope. These two cylinders are centered on $(x, y)$ positions on the bottom plane at $(64,6) \mathrm{Mm}$ and $(-64,-6) \mathrm{Mm}$, with a radius of $20 \mathrm{Mm}$. Within them, $H_{1}$ is constant in the chromosphere and decays rapidly with height above the approximate location of the transition region:

$$
H_{1}= \begin{cases}f(t) c_{1} e^{-\left(\left(z-z_{h}\right) / H_{m}\right)^{2}} & \text { if } z>z_{h} \\ f(t) c_{1} & \text { if } z \leqslant z_{h}\end{cases}
$$

where $\quad c_{1}=10^{-2} \mathrm{erg} \mathrm{cm}^{-3} \mathrm{~s}^{-1}, \quad z_{h}=5 \mathrm{Mm}, \quad$ and $H_{m}=3.16 \mathrm{Mm}$. This final stage is simulated for a total of over four hours, and it is this stage that is presented in what follows.

\section{RESULTS}

As chromospheric plasma gets evaporated into the corona, the lower part of the flux rope slowly evolves into a thermally unstable situation due to the dominating radiative cooling. After about 100 minutes of gradual evolution, we then witness the runaway cooling stage when the decrease of temperature amplifies the radiation, leading to the formation of dynamic blobs and threads. To get an overall impression of the evolution, we quantify the temporal evolution of the mass, the average density, as well as the average velocity of all prominence plasma and all coronal plasma in the computational box (see Figures 2(a) and (b)). In this quantification, prominence plasma has a number density higher than $10^{10} \mathrm{~cm}^{-3}$, a temperature lower than $20,000 \mathrm{~K}$, and an altitude higher than $8 \mathrm{Mm}$. The change in local density and temperature conditions ultimately reaches the threshold to trigger thermal instability, with plasma condensations forming at a rapid pace after 80 minutes, accompanied by a decrease in coronal mass. At a time of 134.5 minutes, the mass of the prominence reaches a maximal $3.1 \times 10^{13} \mathrm{~g}$, and then starts decreasing as some blobs have by now fallen into the chromosphere. After 164.5 minutes, the mass of the prominence settles and starts to oscillate around an average value of $1.65 \times 10^{13} \mathrm{~g}$, while the mass of the coronal volume reaches a stable value of $8.3 \times 10^{14} \mathrm{~g}$. The number density of the prominence instantly 

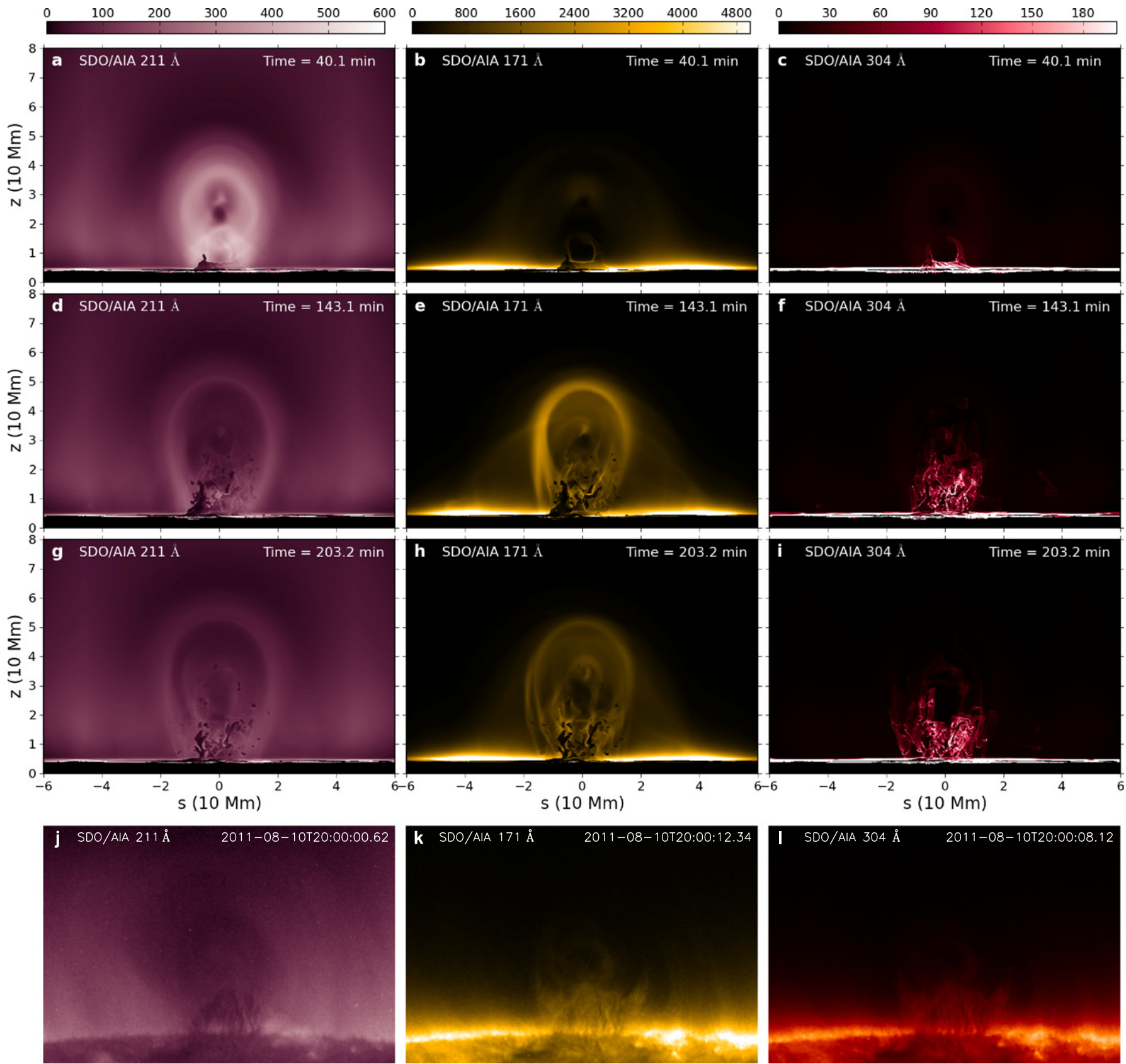

Figure 5. Synthetic EUV images of the forming prominence viewed along the axis of the prominence and comparison with observations. Synthetic SDO/AIA EUV images of the simulated prominence at wavebands 211, 171, and $304 \AA$, at times of (a)-(c) 40.1 minutes, (d)-(f) 143.1 minutes, and (g)-(i) 203.2 minutes; and (j)-(l) SDO/AIA observations on a real prominence and its cavity. Supplementary Movie 1 shows the temporal evolution of these synthetic views.

(An animation of this figure is available.)

adopts a value consistent with observations, as it oscillates around a mean value of $1.88 \times 10^{10} \mathrm{~cm}^{-3}$. The average temperatures of the prominence and the corona are $17,500 \mathrm{~K}$ and $1,660,000 \mathrm{~K}$, respectively. For the entire 80 minutes following, we see lots of dynamics in the prominence as a whole, but the drainage of prominence mass into the chromosphere is balanced by newborn prominence material in continuously forming condensations.

If we concentrate on the later stable phase after 164.5 minutes, the average vertical velocity of the prominence plasma is $-4.87 \mathrm{~km} \mathrm{~s}^{-1}$, showing that the prominence plasma is generally descending at a speed much slower than free-fall speed, which is consistent with observations (Liu et al. 2012). To understand the mass flows, we quantify the mass flux through a horizontal plane at the bottom of the corona, including the total mass flux as well as contributions from prominence plasma, coronal plasma, and plasma in the prominence-corona transition region (PCTR) (Figure 2(c)). Because of the chromospheric evaporation, the upward mass flux increases in the first 7 minutes and then decreases, impeded by the build-up of a high gas pressure inside the flux rope. As soon as the condensations begin, the evaporating mass flow increases slowly and then gradually settles at around $3.1 \times 10^{12} \mathrm{~g} \mathrm{~s}^{-1}$. Prominence plasma with surrounding PCTR 

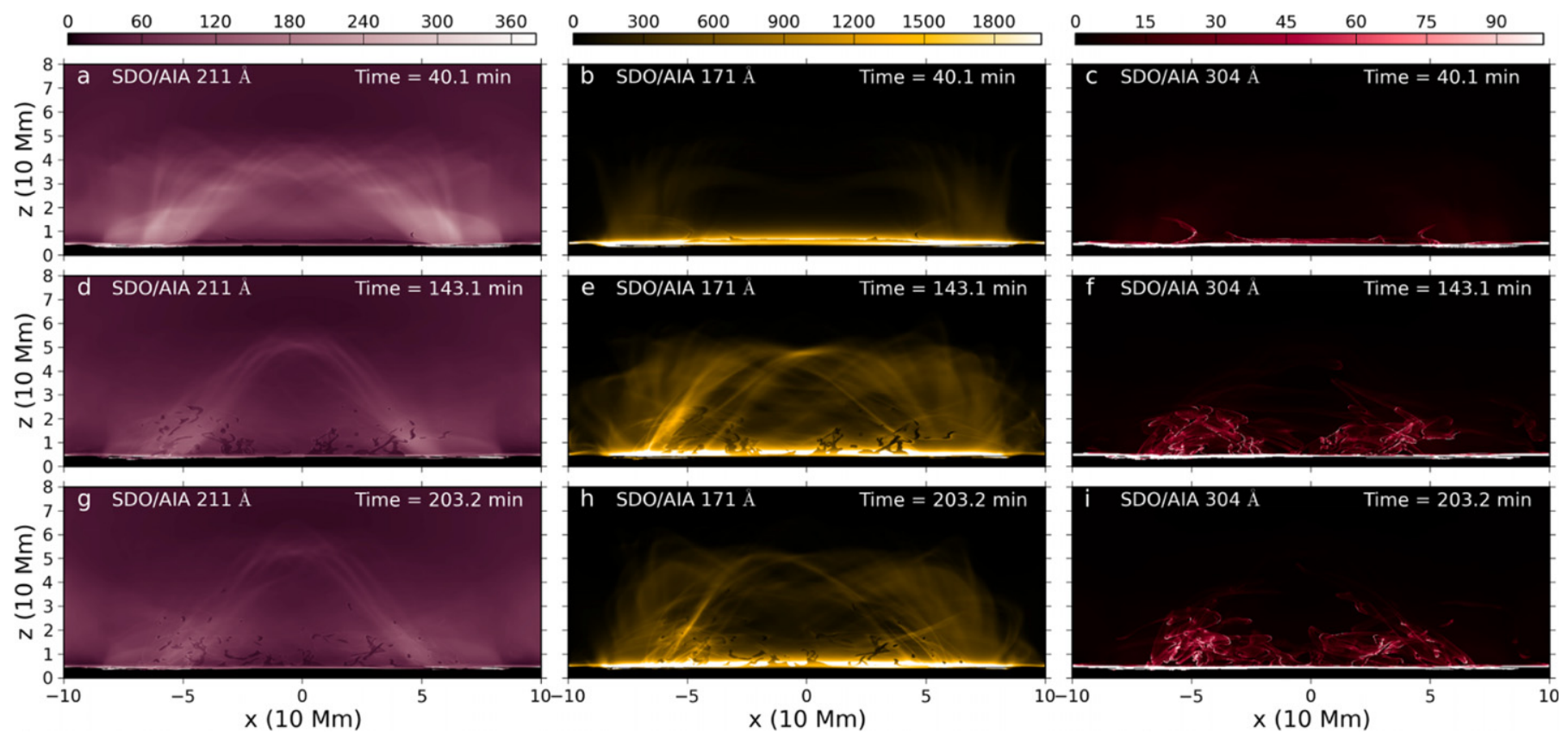

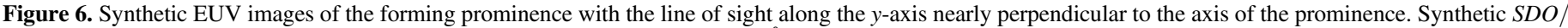

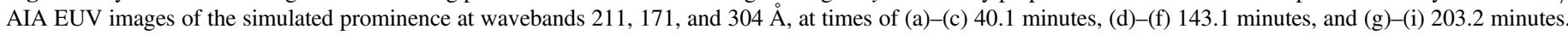
Supplementary Movie 2 shows the temporal evolution of these synthetic views.

(An animation of this figure is available.)

plasma starts to fall down through this plane back into the chromosphere after 110 minutes. The corresponding mass fluxes of prominence plasma and PCTR plasma oscillate with time and eventually stabilize at around $-10^{12} \mathrm{~g} \mathrm{~s}^{-1}$ and $-2.1 \times 10^{12} \mathrm{~g} \mathrm{~s}^{-1}$, respectively. The total mass flux oscillates around zero, implying a dynamical equilibrium where a steady cycling of mass is fully operational. We can draw an analogy with the water cycle on Earth, since we have solar chromospheric plasma that evaporates from the footpoints of the flux rope to reach higher altitudes in the corona, where radiative condensations accumulate mass in prominence blobs that ultimately fall back to the chromosphere.

The most striking finding of our model is the extremely dynamic and intrinsically fragmented appearance of the prominence matter, from its first instant of formation throughout its steady plasma cycle. The prominence plasma is born in thin threads and blobs distributed over different places in the flux rope as visualized for a particular instant in Figure 3(a). Some of the blobs appear and then reside in the central dipped region of fairly left-right symmetrical, twisted field lines. Some blobs form and then surf along the shallow elbows of asymmetrically twisted field lines. A few blobs also pop up and then seemingly follow arched regions of weakly twisted field lines. Although every condensation has a different size and shape, the cross sections perpendicular to their long axis are roughly round. The diameter of these cross sections ranges from 1000 to $1800 \mathrm{~km}$. A typical variation of the temperature, density, and velocity field across an individual blob is shown for the thread in the enlarged panel of Figure 3(b). There are typically shearing flows at both sides of the thread (Figure 3(c)). These flows themselves influence the dynamical evolution of the thread, and the velocity difference across the thread reaches $115 \mathrm{~km} \mathrm{~s}^{-1}$ with local Alfvén Mach number up to 1 .
We already mentioned that the average vertical velocity in prominence matter is a few kilometers per second downwards. To see how the complex plasma motions of prominence fragments interact with the magnetic field, we use particle tracers to follow fluid elements. At a time of 143.1 minutes, we position massless and uncharged particles, which move passively with the local velocity field, in the cores of prominence threads, where plasma density is higher than $2 \times 10^{10} \mathrm{~cm}^{-3}$ with temperature lower than $20,000 \mathrm{~K}$, and plasma $\beta$ (the ratio between gas and magnetic pressure) ranges from 0.2 to 0.25 . Each particle evolution involves integrating the advection equation with velocity information interpolated from the MHD run. In this integration process we introduce particle time steps different from the fluid time steps (limited by the Courant-Friedrichs-Lewy condition) adopted in the time integration of the MHD equations: within one MHD fluid time step, the particles evolve more than one particle time step since particles are restricted to travel a distance shorter than the size of a grid cell in one particle time step. To evaluate the velocity of a particle at a particle time between two fluid time steps, we need both spatial and temporal interpolations of the velocity data from the MHD run. We first get two velocities at the particle position by linear spatial interpolation of the fluid velocity, one for each fluid time step. Then we do another linear interpolation in time between these two velocities to quantify the instantaneous local velocity field. We solve the equation of motion of the particle itself using a fourth-order Runge-Kutta scheme with adaptive step size. As the magnetic field is frozen-in the plasma due to the high conductivity in the solar atmosphere, these tracer particles allow us to disentangle the movement of individual magnetic field lines and the fluid dynamics. We find that, generally, those condensations that form and collect in the dips of helical magnetic field lines move downward under gravity, dragging the field lines down to make 
the dips deeper (Figure 4). Note that the translucent yellow field line at a time of 143.1 minutes is bent by the falling blob to become the green field line at a later time of 214.7 minutes. A typical descending speed is about $6 \mathrm{~km} \mathrm{~s}^{-1}$ for the central condensations. On the other hand, condensations forming in the legs of arched magnetic field lines slip down along the field lines without significant deformation of these field lines. The heated arcade loops of the flux rope rise against the overlying constraining magnetic arcade, and this also leads to interchange motions with rising flux rope loops and sinking coronal loops in higher regions above the prominence.

To validate our model, we have made synthetic observations on our simulations with technique details described in Xia et al. (2014a), for direct comparison with those from the Solar Dynamics Observatory Atmospheric Imaging Assembly (AIA) instrument. Synthetic EUV images are generated from different viewing angles through the AIA wavebands at 211, 193, 171, and $304 \AA$, which have main contribution temperatures around $1.8,1.5,0.8$, and $0.08 \mathrm{MK}$, respectively. To mimic absorption of background EUV emission by the prominence plasma, we exclude emission coming from behind prominence plasma with a density higher than $2 \times 10^{10} \mathrm{~cm}^{-3}$. To view along the axis of the prominence, we select a horizontal line of sight that deviates from the $x$-axis by 8.4 . Viewing along the axis of the prominence, the flux rope region becomes brighter and reaches a maximum sequentially in the 211,193 , and $171 \AA$ EUV channels at times of 55.8 minutes, 78.7 minutes, and 95.9 minutes, respectively. At a time of 40.1 minutes, bright hornlike structures in the $304 \AA$ image protrude from the chromosphere, showing that the prominence plasma first forms at low altitudes. Later on, dynamic dark clumps and threads - the dense cores of the condensations-appear in the lower part of the flux rope in the 211 and $171 \AA$ hot channels, while they are bright in the $304 \AA$ cool channel (Figure 5). Smaller clumps tend to form at higher altitudes and fall down into the chromosphere along curved trajectories. Larger clumps and threads often present vertical pillars collected in the dipped region of the flux rope. A dark coronal cavity enclosed by a bright elliptical loop appears above the dark condensations in hot channels, with higher contrast in hotter channels. The coronal cavity expands slowly with time and reaches a height of $54 \mathrm{Mm}$ at the end of our simulation. In the bottom row of Figure 5, we plot AIA observations of a real prominence on 2011 August 10 for direct comparison. Our modeled prominence closely resembles the real observed one. We prepared a movie (Supplementary Movie 1) to show the temporal evolution of the axial view of the modeled prominence in AIA 211, 193, 171, and $304 \AA$ channels. While observations on solar prominences are limited to one or two viewing angles, we can observe our simulated prominence from any viewing angle. When we put the line of sight along the $y$-axis, we witness the formation of the prominence from the flank. From this vantage point, the weak EUV brightness in the initial corona is quite homogeneous until the localized heating induces sudden brightening of the flux rope where many thin helical loops appear prominently in the 211 and $193 \AA$ images. At a time of 40.1 minutes, two oblique condensations, far from each other, grow in the dipped portions of asymmetric twisted loops. In fact, these widely separated threads are those that resemble the horns in the axial view. At a time of 95.9 minutes, two long condensed threads, about $40 \mathrm{Mm}$ long in $304 \AA$, stretch horizontally across helical field lines at altitudes of about $20 \mathrm{Mm}$. Then numerous fragmented condensations appear and cluster in several places. They follow different curved trajectories of varying shape and size. Many of them slip into the left and right footpoints of the flux rope, while others linger in the central dipped regions (see Supplementary Movie 2).

\section{CONCLUSIONS AND DISCUSSION}

The model described above demonstrates the physical mechanism responsible for the formation of prominences in realistic 3D coronal environments and self-consistently explains the vertical flows in thin prominence threads, which reveal the plasma circulation of long-lived prominences. Their longevity is in analogy with the water cycle on Earth, where plasma evaporates from the chromosphere into the corona, in situ condensations happen continuously to form threads by runaway cooling, while prominence threads descend and eventually fall back to the chromosphere, dragging along the mass-loaded magnetic field lines. This offers completely new insights on how prominence matter gets recycled between chromospheric and coronal heights, and revolutionizes all previous models where a more static, large-scale structure to model prominences is adopted. Since many prominences erupt into interplanetary space at the end of their lives, they are vital ingredients in coronal mass ejections (CMEs), which may have severe impact on the terrestrial space environment. To better understand prominence eruption and CMEs, realistic models of prominences are needed. Our model represents a milestone in constructing such models and can now be used to initiate and study the eruption phase in detail.

C.X. wants to thank FWO (Research Foundation Flanders) for the award of postdoctoral fellowship and thank Oliver Porth, Yang Guo, and Junjie Yi for discussions. This research was supported by FWO and the Interuniversity Attraction Poles Programme by the Belgian Science Policy Office (IAP P7/08 CHARM). The simulations were conducted on the VSC (Flemish Supercomputer Center funded by Hercules foundation and Flemish government) and SuperMUC supercomputers (provided by PRACE resources in project pr87di).

\section{REFERENCES}

Antiochos, S. K., MacNeice, P. J., Spicer, D. S., \& Klimchuk, J. A. 1999, ApJ, 512, 985

Bak-Stȩślicka, U., Gibson, S. E., Fan, Y., et al. 2013, ApJL, 770, L28

Berger, T., Testa, P., Hillier, A., et al. 2011, Natur, 472, 197

Berger, T. E., Liu, W., \& Low, B. C. 2012, ApJL, 758, L37

Berger, T. E., Shine, R. A., Slater, G. L., et al. 2008, ApJL, 676, L89

Berger, T. E., Slater, G., Hurlburt, N., et al. 2010, ApJ, 716, 1288

Blokland, J. W. S., \& Keppens, R. 2011, A\&A, 532, A93

Bommier, V., Landi Degl'Innocenti, E., Leroy, J.-L., \& Sahal-Brechot, S. 1994, SoPh, 154, 231

Čada, M., \& Torrilhon, M. 2009, JCoPh, 228, 4118

Chae, J., Ahn, K., Lim, E.-K., Choe, G. S., \& Sakurai, T. 2008, ApJL, 689, L73

Chiu, Y. T., \& Hilton, H. H. 1977, ApJ, 212, 873

Dedner, A., Kemm, F., Kröner, D., et al. 2002, JCoPh, 175, 645

Engvold, O. 1976, SoPh, 49, 283

Field, G. B. 1965, ApJ, 142, 531

Fuller, J., Gibson, S. E., de Toma, G., \& Fan, Y. 2008, ApJ, 678, 515

Gibson, S. E., Kucera, T. A., Rastawicki, D., et al. 2010, ApJ, 724, 1133

Harten, A., Lax, P. D., \& van Leer, B. 1983, SIAMR, 25, 35

Hillier, A., Isobe, H., Shibata, K., \& Berger, T. 2011, ApJL, 736, L1

Hillier, A., \& van Ballegooijen, A. 2013, ApJ, 766, 126

Kaneko, T., \& Yokoyama, T. 2015, ApJ, 806, 115 
Karpen, J. T., Antiochos, S. K., Hohensee, M., Klimchuk, J. A., \& MacNeice, P. J. 2001, ApJL, 553, L85

Keppens, R., Meliani, Z., van Marle, A. J., et al. 2012, JCoPh, 231, 718

Keppens, R., \& Xia, C. 2014, ApJ, 789, 22

Keppens, R., Xia, C., \& Porth, O. 2015, ApJL, 806, L13

Khomenko, E., Díaz, A., de Vicente, A., Collados, M., \& Luna, M. 2014, A\&A, 565, A45

Kuperus, M., \& Raadu, M. A. 1974, A\&A, 31, 189

Leighton, R. B. 1964, ApJ, 140, 1547

Leroy, J. L., Bommier, V., \& Sahal-Brechot, S. 1983, SoPh, 83, 135

Liu, W., Berger, T. E., \& Low, B. C. 2012, ApJL, 745, L21

Low, B. C., \& Zhang, M. 2004, ApJ, 609, 1098

Luna, M., Karpen, J. T., \& DeVore, C. R. 2012, ApJ, 746, 30

Meyer, C. D., Balsara, D. S., \& Aslam, T. D. 2012, MNRAS, 422, 2102
Orozco Suárez, D., Asensio Ramos, A., \& Trujillo Bueno, J. 2014, A\&A, 566, A46

Parker, E. N. 1953, ApJ, 117, 431

Porth, O., Xia, C., Hendrix, T., Moschou, S. P., \& Keppens, R. 2014, ApJ, 214, 4

Ryutova, M., Berger, T., Frank, Z., Tarbell, T., \& Title, A. 2010, SoPh, 267, 75

Terradas, J., Soler, R., Luna, M., Oliver, R., \& Ballester, J. L. 2015, ApJ, 799, 94

Townsend, R. H. D. 2009, ApJ, 181, 391

van Ballegooijen, A. A., \& Martens, P. C. H. 1989, ApJ, 343, 971

Wang, Y.-M., \& Stenborg, G. 2010, ApJL, 719, L181

Xia, C., Chen, P. F., \& Keppens, R. 2012, ApJL, 748, L26

Xia, C., Chen, P. F., Keppens, R., \& van Marle, A. J. 2011, ApJ, 737, 27

Xia, C., Keppens, R., Antolin, P., \& Porth, O. 2014a, ApJL, 792, L38

Xia, C., Keppens, R., \& Guo, Y. 2014b, ApJ, 780, 130 